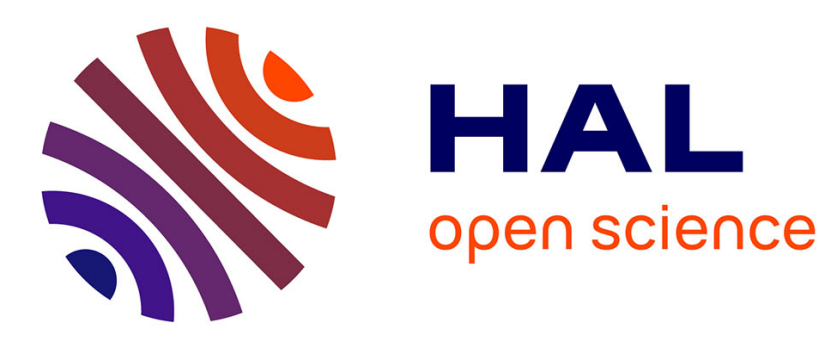

\title{
Robin-Neumann schemes for incompressible fluid-structure interaction
}

Miguel Angel Fernández, Mikel Landajuela, Jimmy Mullaert, Marina Vidrascu

\section{To cite this version:}

Miguel Angel Fernández, Mikel Landajuela, Jimmy Mullaert, Marina Vidrascu. Robin-Neumann schemes for incompressible fluid-structure interaction. Domain Decomposition Methods in Science and Engineering XXII, 2015, Lugano, Switzerland. pp.65-76, 10.1007/978-3-319-18827-0_6 . hal01113088

\section{HAL Id: hal-01113088 \\ https://inria.hal.science/hal-01113088}

Submitted on 4 Feb 2015

HAL is a multi-disciplinary open access archive for the deposit and dissemination of scientific research documents, whether they are published or not. The documents may come from teaching and research institutions in France or abroad, or from public or private research centers.
L'archive ouverte pluridisciplinaire HAL, est destinée au dépôt et à la diffusion de documents scientifiques de niveau recherche, publiés ou non, émanant des établissements d'enseignement et de recherche français ou étrangers, des laboratoires publics ou privés. 


\title{
Robin-Neumann schemes for incompressible fluid-structure interaction
}

\author{
Miguel A. Fernández, Mikel Landajuela, Jimmy Mullaert, and \\ Marina Vidrascu
}

\section{Introduction}

Mathematical problems involving the coupling of an incompressible viscous flow with an elastic structure appear in a large variety of engineering fields (see, e.g., Lombardi et al. [2012], Takizawa and Tezduyar [2012], Païdoussis et al. [2011], Moireau et al. [2012], Heil and Hazel [2011]). This problem is considered here within a heterogenous domain decomposition framework, with the aim of using independent well-suited solvers for the fluid and the solid. One of the main difficulties that have to be faced under this approach is that the coupling can be very stiff. In particular, traditional Dirichlet-Neumann explicit coupling methods, which solve for the fluid (Dirichlet) and for the solid (Neumann) only once per time-step, are unconditionally unstable whenever the amount of added-mass effect in the system is large (see, e.g., Causin et al. [2005]. Förster et al. [2007]). Typically this happens when the fluid and solid densities are close and the fluid domain is slender, as in hemodynamical applications. This explains, in part, the tremendous amount of work devoted over the last decade to the development of alternative coupling paradigms (see, e.g., Fernández [2011] for a review).

In this paper we will review several explicit coupling procedures recently reported in the literature and present some new developments (Section 3.2). The common feature of these methods is that they are based on RobinNeumann transmission conditions, whose nature depends on the thin- or thick-walled character of the structure (see Figure 1).

Inria Paris-Rocquencourt, BP 105, 78153 Le Chesnay Cedex, France

Sorbonne Universités, UPMC Univ. Paris 6, Laboratoire Jacques-Louis Lions, 4 Place Jussieu, 75252 Paris cedex 05, France 

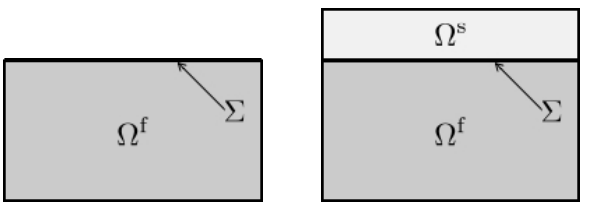

Fig. 1 Fluid-structure configurations for a thin- (left) and a thick-walled structure (right).

\section{Problem formulation}

For the sake of simplicity we consider a low Reynolds regime and assume that the interface undergoes infinitesimal displacements. The fluid is described by the Stokes equations, in a fixed domain $\Omega^{\mathrm{f}} \subset \mathbb{R}^{d}(d=2,3)$, and the structure by the linear (possibly damped) membrane equations written in the $(d-1)$-manifold $\Omega^{\mathrm{s}} \equiv \Sigma$, which is also the fluid-structure interface (see Figure 1(left)).

The coupled model problem reads therefore as follows: find the fluid velocity $\boldsymbol{u}: \Omega^{\mathrm{f}} \times \mathbb{R}^{+} \rightarrow \mathbb{R}^{d}$, the fluid pressure $p: \Omega^{\mathrm{f}} \times \mathbb{R}^{+} \rightarrow \mathbb{R}$, the solid displacement $\boldsymbol{d}: \Sigma \times \mathbb{R}^{+} \rightarrow \mathbb{R}^{d}$ and the solid velocity $\dot{\boldsymbol{d}}: \Sigma \times \mathbb{R}^{+} \rightarrow \mathbb{R}^{d}$ such that

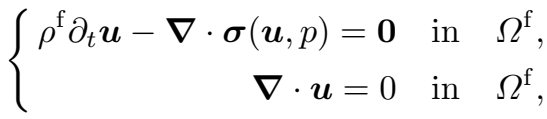

$$
\begin{aligned}
& \left\{\begin{array}{rcc}
\boldsymbol{u}=\dot{\boldsymbol{d}} & \text { on } & \Sigma, \\
\rho^{\mathrm{s}} \epsilon \partial_{t} \dot{\boldsymbol{d}}+\boldsymbol{L}^{\mathrm{e}} \boldsymbol{d}+\boldsymbol{L}^{\mathrm{v}} \dot{\boldsymbol{d}}=-\boldsymbol{\sigma}(\boldsymbol{u}, p) \boldsymbol{n} & \text { on } \quad \Sigma, \\
\dot{\boldsymbol{d}}=\partial_{t} \boldsymbol{d} & \text { on } \quad \Sigma .
\end{array}\right.
\end{aligned}
$$

This system has to be complemented with appropriate initial and (external) boundary conditions, which will be omitted in the following since they are not relevant for the discussion. The symbols $\rho^{\mathrm{f}}$ and $\rho^{\mathrm{s}}$ denote, respectively, the fluid and solid densities, $\epsilon$ is the solid thickness and $\boldsymbol{n}$ stands for the unit normal vector on $\partial \Omega^{\mathrm{f}}$. The fluid Cauchy-stress tensor is given by $\boldsymbol{\sigma}(\boldsymbol{u}, p) \stackrel{\text { def }}{=}-p \boldsymbol{I}+2 \mu \boldsymbol{\varepsilon}(\boldsymbol{u})$, with $\boldsymbol{\varepsilon}(\boldsymbol{u}) \stackrel{\text { def }}{=} \frac{1}{2}\left(\boldsymbol{\nabla} \boldsymbol{u}+\boldsymbol{\nabla} \boldsymbol{u}^{\mathrm{T}}\right)$ and where $\mu$ denotes the fluid dynamic viscosity. Finally, the surface differential operators $\boldsymbol{L}^{\mathrm{e}}$ and $\boldsymbol{L}^{\mathrm{v}}$ describe the membrane elastic and viscous behavior, respectively.

Remark 1. In two spatial dimensions and for the geometrical configuration of Figure 1(left) an example of solid elastic operator is given by $\boldsymbol{L}^{\mathrm{e}} \boldsymbol{d}=$ $\left[0,-c_{1} \partial_{x x} \boldsymbol{d}_{y}+c_{0} \boldsymbol{d}_{y}\right]^{\mathrm{T}}$, where $\boldsymbol{d}=\left[0, \boldsymbol{d}_{y}\right]^{T}$ and $c_{0}, c_{1}>0$ are material dependent parameters. A widely used form of the solid viscous operator is $\boldsymbol{L}^{\mathrm{v}} \dot{\boldsymbol{d}}=\alpha \rho^{\mathrm{s}} \epsilon \dot{\boldsymbol{d}}+\beta \boldsymbol{L}^{\mathrm{e}} \dot{\boldsymbol{d}}$, where $\alpha, \beta>0$ are given parameters. In artery wall modeling, the zeroth-order term, $\alpha \rho^{\mathrm{s}} \epsilon \dot{\boldsymbol{d}}$, describes the dissipative behavior of external tissues (see Moireau et al. [2012]), whereas the differential term, 
$\beta \boldsymbol{L}^{\mathrm{e}} \dot{\boldsymbol{d}}$, corresponds to the Kelvin-Voigt model (see, e.g., Kalita and Schaefer [2008], Valdez-Jasso et al. [2009]).

Remark 2. Though simplified, problem (1)-(2) preserves some of the major numerical difficulties that arise in incompressible fluid-structure interaction.

\section{Explicit coupling schemes}

This section is devoted to the numerical approximation of the coupled problem (1)-(2). In the succeeding text, the symbol $\tau>0$ denotes the timestep size, $t_{n} \stackrel{\text { def }}{=} n \tau$, for $n \in \mathbb{N}$, and $\partial_{\tau} x^{n} \stackrel{\text { def }}{=}\left(x^{n}-x^{n-1}\right) / \tau$ the first order backward difference in time. In addition, the superscript ${ }^{\star}$ is used to indicate zeroth- (i.e., without), first-order or second-order extrapolation from the previous time-steps, namely, $x^{\star}=0$ if $r=0, x^{\star}=x^{n-1}$ if $r=1$ and $x^{\star}=2 x^{n-1}-x^{n-2}$ if $r=2$, where $r$ denotes the extrapolation order.

The methods discussed in this review paper are explicit coupling schemes, in the sense that they enable a decoupled time-marching of the fluid and the solid. Traditional Dirichlet-Neumann explicit coupling procedures, as reported in Algorithm 1, are known to be unconditionally unstable, whenever

Algorithm 1 Dirichet-Neumann explicit coupling scheme For $n \geq 1$ :

1. Fluid step: find $\boldsymbol{u}^{n}: \Omega^{\mathrm{f}} \times \mathbb{R}^{+} \rightarrow \mathbb{R}^{d}$ and $p^{n}: \Omega^{\mathrm{f}} \times \mathbb{R}^{+} \rightarrow \mathbb{R}$ such that

$$
\left\{\begin{array}{rlc}
\rho^{\mathrm{f}} \partial_{\tau} \boldsymbol{u}^{n}-\boldsymbol{\nabla} \cdot \boldsymbol{\sigma}\left(\boldsymbol{u}^{n}, p^{n}\right)=\mathbf{0} & \text { in } \quad \Omega^{\mathrm{f}}, \\
\boldsymbol{\nabla} \cdot \boldsymbol{u}^{n}=0 & \text { in } \quad \Omega^{\mathrm{f}}, \\
\boldsymbol{u}^{n}=\dot{\boldsymbol{d}}^{n-1} & \text { on } \quad \Sigma .
\end{array}\right.
$$

2. Solid step: find $\boldsymbol{d}^{n}: \Sigma \times \mathbb{R}^{+} \rightarrow \mathbb{R}^{d}$ such that

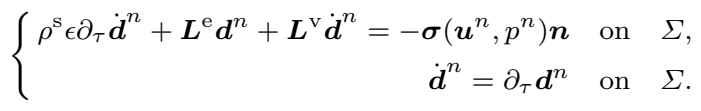

the amount of added-mass effect in the system is large (see, e.g., Causin et al. [2005]). Stability in explicit coupling for incompressible fluid-structure interaction demands a different treatment of the interface coupling conditions $(2)_{1,2}$.

A stable explicit coupling alternative is given by the Robin-Robin methods introduced in Burman and Fernández [2009, 2014], which build on a Nitsche treatment of the interface coupling. A salient feature of these methods is that they do not depend on the thin- or thick-walled nature of the solid. Unfortunately, the explicit treatment of the Nitsche's penalty induces a deterioration 
of the accuracy, which demands restrictive CFL constraints, unless correction iterations with suitable extrapolations are performed (see Burman and Fernández [2014]). Numerical evidence suggests that optimal first-order accuracy can be achieved by using a non-symmetric penalty-free formulation (see [Burman and Fernández, 2014, Section 4.3]). The rigorous stability analysis of the resulting schemes remains, however, an open problem.

\subsection{Robin-Neumann schemes}

The key difficulty is hence the derivation of alternative splitting methods which guarantee stability without compromising accuracy. The RobinNeumann methods proposed in Fernández [2013], Fernández et al. [2013] achieve this purpose. The fundamental ingredient in the derivation of these schemes is the interface Robin consistency featured by the continuous problem (1)-(2). Indeed, from (2) $)_{1,2}$ it follows that

$$
\boldsymbol{\sigma}(\boldsymbol{u}, p) \boldsymbol{n}+\rho^{\mathrm{s}} \epsilon \partial_{t} \boldsymbol{u}=-\boldsymbol{L}^{\mathrm{e}} \boldsymbol{d}-\boldsymbol{L}^{\mathrm{v}} \dot{\boldsymbol{d}} \quad \text { on } \quad \Sigma,
$$

which can be viewed as a Robin-like boundary condition for the fluid. Hence, instead of performing the fluid solid time splitting in terms of $(2)_{1,2}$ as in Algorithm 1, we consider (3) and $(2)_{2}$. The resulting schemes are detailed in Algorithm 2.

Algorithm 2 Robin-Neumann explicit coupling schemes (from Fernández et al. [2013]).

For $n \geq r+1$ :

1. Fluid step: find $\boldsymbol{u}^{n}: \Omega^{\mathrm{f}} \times \mathbb{R}^{+} \rightarrow \mathbb{R}^{d}$ and $p^{n}: \Omega^{\mathrm{f}} \times \mathbb{R}^{+} \rightarrow \mathbb{R}$ such that

$$
\left\{\begin{aligned}
\rho^{\mathrm{f}} \partial_{\tau} \boldsymbol{u}^{n}-\boldsymbol{\nabla} \cdot \boldsymbol{\sigma}\left(\boldsymbol{u}^{n}, p^{n}\right)=\mathbf{0} & \text { in } \quad \Omega^{\mathrm{f}}, \\
\boldsymbol{\nabla} \cdot \boldsymbol{u}^{n}=0 & \text { in } \quad \Omega^{\mathrm{f}}, \\
\boldsymbol{\sigma}\left(\boldsymbol{u}^{n}, p^{n}\right) \boldsymbol{n}+\frac{\rho^{\mathrm{s} \epsilon}}{\tau} \boldsymbol{u}^{n}=\frac{\rho^{\mathrm{s}} \epsilon}{\tau} \dot{\boldsymbol{d}}^{n-1}-\boldsymbol{L}^{\mathrm{e}} \boldsymbol{d}^{\star}-\boldsymbol{L}^{\mathrm{v}} \dot{\boldsymbol{d}}^{\star} & \text { on } \quad \Sigma .
\end{aligned}\right.
$$

2. Solid step: find $\boldsymbol{d}^{n}: \Sigma \times \mathbb{R}^{+} \rightarrow \mathbb{R}^{d}$ such that

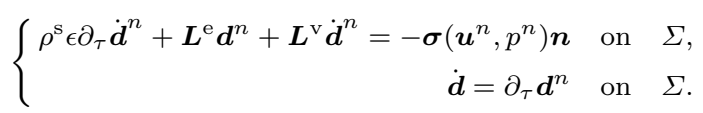

Algorithm 2 completely uncouples the fluid and solid time-marchings. This is achieved via the explicit Robin condition $(4)_{3}$ derived from (3). Note that only the solid inertial effects are implicitly treated in $(4)_{3}$, this is enough to guarantee added-mass free stability. It is also worth noting that, from $(5)_{1}$, 
the explicit Robin condition $(4)_{3}$ can be reformulated as

$$
\boldsymbol{\sigma}\left(\boldsymbol{u}^{n}, p^{n}\right) \boldsymbol{n}+\frac{\rho^{\mathrm{s}} \epsilon}{\tau} \boldsymbol{u}^{n}=\frac{\rho^{\mathrm{s}} \epsilon}{\tau}\left(\dot{\boldsymbol{d}}^{n-1}+\tau \partial_{\tau} \dot{\boldsymbol{d}}^{\star}\right)+\boldsymbol{\sigma}\left(\boldsymbol{u}^{\star}, p^{\star}\right) \boldsymbol{n} \quad \text { on } \quad \Sigma .
$$

The advantage of this new expression is its intrinsic character, in the sense that it avoids extrapolations of the solid viscoelastic terms within the fluid solver.

Remark 3. It should be noted that the implicit treatment of the solid-damping term $\boldsymbol{L}^{\mathrm{v}}$ in (4), as advocated in Guidoboni et al. [2009], Lukáčová-Medvid'ová et al. [2013], Bukač et al. [2013], yields a coupling scheme which is not explicit: it is semi-implicit. Moreover, the resulting solution procedure is not partitioned either, since the solid viscous contribution $\boldsymbol{L}^{\mathrm{v}}$ has to be integrated within the fluid solver.

Theoretical results on the stability and accuracy of Algorithm 2 have been reported in Fernández [2013] and Fernández et al. [2013]. A fundamental ingredient in the analysis is the fact that Algorithm 2 can be viewed as a fully implicit scheme with the following perturbed kinematic constraint

$$
\boldsymbol{u}^{n}=\dot{\boldsymbol{d}}^{n}+\frac{\tau}{\rho^{\mathrm{s}} \epsilon}\left[\boldsymbol{L}^{\mathrm{e}}\left(\boldsymbol{d}^{n}-\boldsymbol{d}^{\star}\right)+\boldsymbol{L}^{\mathrm{v}}\left(\dot{\boldsymbol{d}}^{n}-\dot{\boldsymbol{d}}^{\star}\right)\right] \quad \text { on } \quad \Sigma .
$$

The stability and the accuracy of Algorithm 2 are hence driven by the impact of this perturbation (i.e., the last term of (6)) on the stability and accuracy of the underlying implicit coupling scheme. Unconditional energy stability can be proved for $r=0$ and $r=1$. The scheme with $r=2$ is energy stable under a CFL-like condition. As regards accuracy, the error analysis shows that the splitting error induced by the kinematic perturbation (6) scales as $\mathcal{O}\left(\tau^{2^{r-1}}\right)$. Thus, Algorithm 2 with $r=1$ or $r=2$ yields an overall optimal first-order time-accuracy $\mathcal{O}(\tau)$ in the energy-norm, while a sub-optimal time convergence rate $\mathcal{O}\left(\tau^{\frac{1}{2}}\right)$ is expected for the scheme with $r=0$.

Remark 4. In the particular case of an undamped thin-walled solid (i.e., $\boldsymbol{L}^{\mathrm{v}}=$ 0), Algorithm 2 with $r=0$ yields the splitting scheme reported in Guidoboni et al. [2009], which is known to deliver very poor accuracy (see Fernández [2013], Fernández et al. [2013] and the example below).

We conclude this section with a numerical illustration based on the balloon-like example proposed in [Küttler et al., 2006, Section 7.1] and using a non-linear version of (1)-(2). This type of problems involving fully enclosed fluids cannot be solved using Algorithm 1 (or iterative variants) due to the constraint enforced by the fluid incompressibility on the interface solid velocity (unless it is directly prescribed in the solid solver, see Küttler et al. [2006]). Figure 2(left) presents some snapshots of the fluid velocity magnitude in the deformed configuration obtained with a non-linear version of Algorithm 2 $(r=1$ and $\tau=0.05)$. The fluid equations are discretized in space with 
$\mathbb{Q}_{1} / \mathbb{Q}_{1}$ finite elements and a SUPG/PSPG stabilized formulation. Quadrilateral MITC4 (locking-free) shell elements are considered for the structure (see, e.g., Chapelle and Bathe [2011]). For comparison purposes, Figure 2(right) shows the maximal displacement magnitude on the interface obtained with Algorithm 2 and the implicit coupling scheme. Algorithm 2 with $r=1$ or $r=2$ provides numerical solutions close to the implicit scheme. The superior accuracy of the variant with $r=2$, induced by the second-order extrapolation in (4), is clearly noticeable. On the contrary, Algorithm 2 with $r=0$ (see Remark 4) yields an extremely poor approximation. This is a clear indication of the $\mathcal{O}\left(\tau^{\frac{1}{2}}\right)$-loss in the accuracy of the scheme predicted by the error analysis.
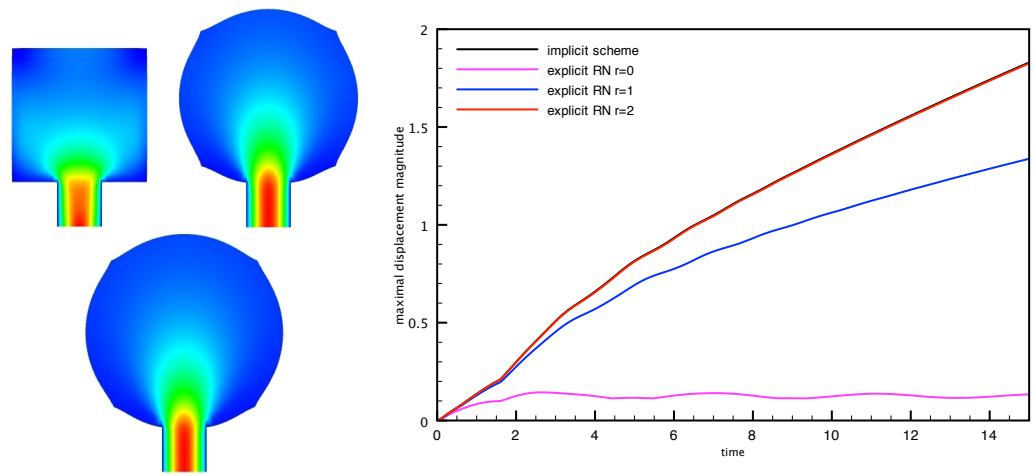

Fig. 2 Left: Snapshots of the fluid velocity magnitude in the deformed configuration at $t=0.15,7.5,15$ (Algorithm 2 with $r=1$ and $\tau=0.05$ ). Right: Comparison of the solid displacements vs. time obtained with Algorithm 2 and the implicit scheme $(\tau=0.05)$.

\subsection{Second-order accuracy}

So far no explicit stable second-order time-accurate scheme is known for general fluid-structure interaction. For purely elastic thin-structures, some attempts have been presented in Lukáčová-Medvid'ová et al. [2013] by combining a Strang operator splitting approach with the ideas reported in Guidoboni et al. [2009]. Though the accuracy of the splitting is improved, second-order time-accuracy is still not achieved.

In this section we show how the Robin-Neumann explicit coupling paradigm of Section 3.1 can be adapted to deliver second-order time-accuracy. This is achieved by combining a Crank-Nicholson time-stepping in both the fluid and the solid subproblems, with an enhanced time-discretization of (3) based on either second-order extrapolation or defect-correction iterations. It is worth noting that this strategy for enhancing accuracy might lead to stability prob- 
lems when applied to other explicit coupling paradigms (see, e.g., Burman and Fernández [2014]).

The resulting schemes are displayed in Algorithm 3, where $K \geq 0$ denotes the number of correction iterations and $x^{n-\frac{1}{2}, k} \stackrel{\text { def }}{=}\left(x^{n, k}+x^{n-1}\right) / 2$ stands for the midpoint between the previous value $x^{n-1}$ and the $k$-stage corrected one $x^{n, k}$.

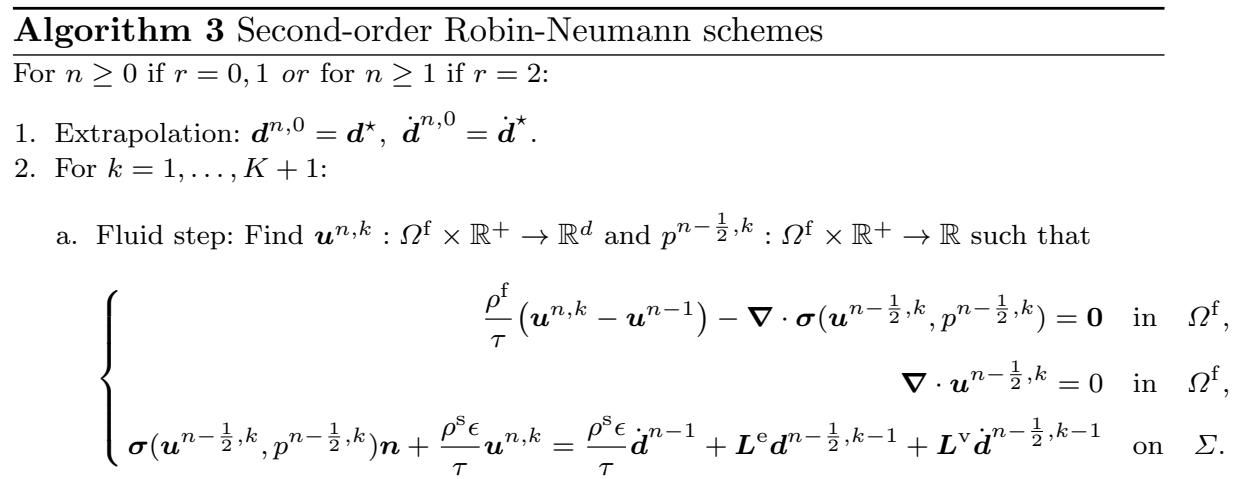

b. Solid step: Find $\boldsymbol{d}^{n, k}: \Sigma \times \mathbb{R}^{+} \rightarrow \mathbb{R}^{d}$ such that

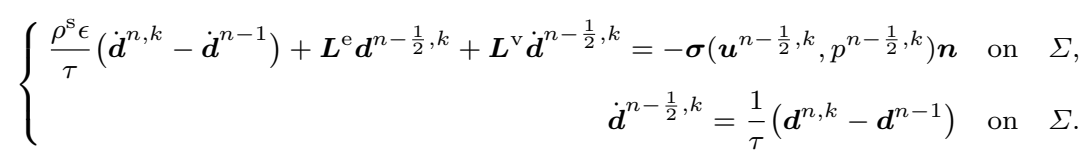

3. Solution update: $\boldsymbol{u}^{n}=\boldsymbol{u}^{n, K+1}, p^{n-\frac{1}{2}}=p^{n-\frac{1}{2}, K+1}, \boldsymbol{d}^{n}=\boldsymbol{d}^{n, K+1}, \dot{\boldsymbol{d}}^{n}=\dot{\boldsymbol{d}}^{n, K+1}$.

Similarly to Algorithm 2, Algorithm 3 with $K=0$ can be regarded as interface kinematic perturbations of an underlying second-order implicit scheme. Hence, in order to achieve overall second-order time-accuracy, two approaches are investigated:

1. $r=1$ and $K>0$ : Recall that the consistency errors induced by the kinematic perturbations with $r=1$ scale as $\mathcal{O}(\tau)$. Thus, after $K>0$ defect-corrections the perturbation of the kinematic constraint scales as $\mathcal{O}\left(\tau^{K+1}\right)$. Hence, in order to retrieve second-order time-accuracy $K=1$ will be enough.

2. $r=2$ and $K=0$ (genuine explicit scheme): Since the consistency error induced with $r=2$ scales as $\mathcal{O}\left(\tau^{2}\right)$, no defect-correction is needed.

To give some insight into the stability properties of Algorithm 3, we consider a simplification of the model problem (1)-(2) at hand (see, e.g., Causin et al. [2005], Badia et al. [2008]). Specifically, we take $\Omega^{\mathrm{f}}=[0, L] \times[0, R] \subset \mathbb{R}^{2}$, $\Sigma=\{y=R\}$, the solid operators of Remark 1 and $\mu=0$ (potential fluid). In this framework the following proposition holds. 
Proposition 1. Take $K=0$ (no defect-correction) in Algorithm 3 and write $\boldsymbol{d}_{y}^{n}=\sum_{i=1}^{\infty} \boldsymbol{d}_{y, i}^{n} \phi_{i}$ where we consider the orthonormal basis on $L_{0}^{2}(\Sigma)$ given by $\left\{\phi_{i}(x)=\sqrt{2 / L} \sin (i \pi x / L)\right\}_{i=1}^{\infty}$. Under the problem setting described in the previous paragraph, we have:

1. If $r=0$ or $r=1, \quad\left|\boldsymbol{d}_{y, i}^{n}\right| \underset{n \rightarrow+\infty}{\longrightarrow} 0 \quad \forall i \in\{1, \ldots, \infty\}$.

2. If $r=2, \quad\left|\boldsymbol{d}_{y, i}^{n}\right| \underset{n \rightarrow+\infty}{\longrightarrow} 0$ with $i \in\{1, \ldots, \infty\}$ provided

$$
\left\{\begin{array}{l}
4 a_{i} b_{i}+4 b_{i}^{2} \tau+4 d_{i} b_{i} \tau^{2}+\frac{d_{i}^{2}\left(-2 \mu_{i} \rho^{\mathrm{f}}+\epsilon \rho^{\mathrm{s}}\right)}{\epsilon \rho^{\mathrm{s}}} \tau^{3} \geq 0 \\
4 e_{i} \epsilon \rho^{\mathrm{s}}\left(b_{i}+d_{i} \tau\right)\left(4 a_{i}+d_{i} \tau^{2}+4 \tau b_{i}\right)-4 d_{i} \tau e_{i}^{2} \\
\quad-16 \epsilon \rho^{\mathrm{s}} \tau\left(b_{i}+d_{i} \tau\right)^{2}\left(a_{i} \epsilon \rho^{\mathrm{s}}+2 b_{i} \mu_{i} \rho^{\mathrm{f}} \tau\right) \geq 0
\end{array}\right.
$$

where $a_{i}=\mu_{i} \rho^{\mathrm{f}}+\epsilon \rho^{\mathrm{s}}, b_{i}=\beta c_{1} \lambda_{i}+\alpha \epsilon \rho^{\mathrm{s}}, d_{i}=c_{0}+c_{1} \lambda_{i}, e_{i}=4 a_{i} \epsilon \rho^{\mathrm{s}}+$ $\tau\left(b_{i} \epsilon \rho^{\mathrm{s}}+2 d_{i} \mu_{i} \rho^{\mathrm{f}} \tau\right)$ and $\mu_{i}=\frac{L}{i \pi \tanh \left(\frac{i \pi R}{L}\right)}, \lambda_{i}=\frac{i^{2} \pi^{2}}{L^{2}}$ are the eigenvalues with respect to $\phi_{i}$ of the Neumann-to-Dirichlet map and $\partial_{x x}$ operators.

Proposition 1 establishes that whenever the Fourier series expansion of $\boldsymbol{d}_{y}^{n}$ is truncated (i.e., whenever the spatial discretization is fixed) the solution of Algorithm 3 with $K=0$, under the above assumptions, is unconditionally stable with zeroth- and first-order extrapolations. For $r=2$, the conditions (7) might be too restrictive since they do not explicitly take into account the effect of the spatial discretization step $h$.

In order to numerically illustrate the accuracy and stability of Algorithm 3, we consider the two-dimensional example of Fernández et al. [2013]. To pro-
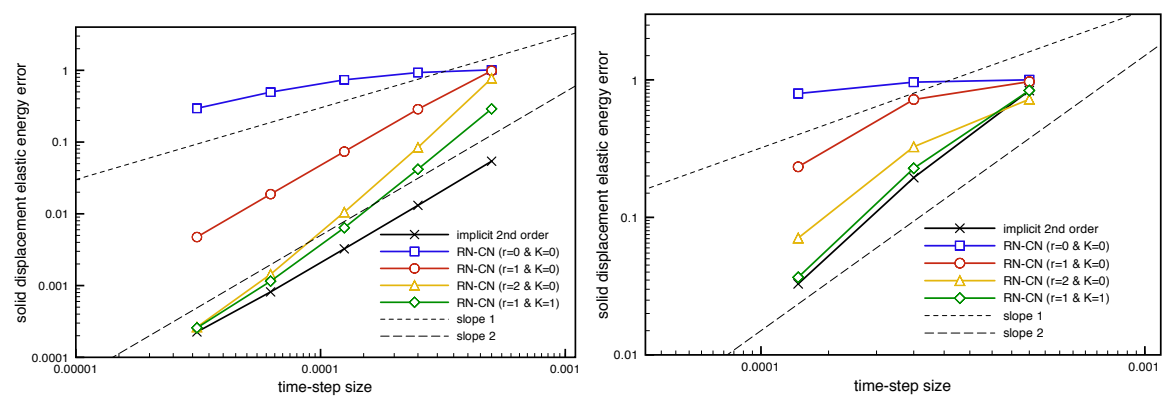

Fig. 3 Left: displacement convergence history in time with $h=10^{-1} / 4$ fixed. Right: displacement convergence history in time with $h=\mathcal{O}\left(\tau^{2}\right)$.

vide evidence on the $\mathcal{O}\left(h+\tau^{2}\right)$ convergence behavior for the first and second order extrapolated variants, Figure 3 (left) reports the time-convergence history, with $h=10^{-1} / 4$ fixed, of the solid displacement at time $t=0.015$, in the relative elastic energy-norm, obtained with Algorithm 3 and a fully 
implicit second-order scheme. The reference solution has been generated using the implicit scheme with $\tau=10^{-6}$ and the same $h$. The $h$-uniformity is guaranteed by Figure 3 (right) were we have refined both in time and space according $h=\mathcal{O}\left(\tau^{2}\right)$. The reference solution has been now obtained with $\tau=10^{-6}$ and $h=3 \times 10^{-3}$.

\subsection{Coupling with thick-walled structures}

In this section we briefly describe the extension of the Robin-Neumann explicit coupling paradigm of Algorithm 2 to the case of the coupling with thick-walled structures (see Figure 1(right)). Thus, in the coupled problem (1)-(2), the relations (2) are replaced by the linear elastodynamics equations

$$
\left\{\begin{aligned}
\rho^{\mathrm{s}} \partial_{t} \dot{\boldsymbol{d}}+\alpha \rho^{\mathrm{s}} \dot{\boldsymbol{d}}-\nabla \cdot \boldsymbol{\Pi}(\boldsymbol{d}, \dot{\boldsymbol{d}})=\mathbf{0} & \text { in } \quad \Omega^{\mathrm{s}}, \\
\dot{\boldsymbol{d}}=\partial_{t} \boldsymbol{d} & \text { in } \quad \Omega^{\mathrm{s}},
\end{aligned}\right.
$$

together with the kinematic and kinetic coupling conditions

$$
\left\{\begin{array}{rcc}
\boldsymbol{u}=\dot{\boldsymbol{d}} & \text { on } \quad \Sigma, \\
\boldsymbol{\Pi}(\boldsymbol{d}, \dot{\boldsymbol{d}}) \boldsymbol{n}^{\mathrm{s}}=-\boldsymbol{\sigma}(\boldsymbol{u}, p) \boldsymbol{n} & \text { on } \quad \Sigma .
\end{array}\right.
$$

Here, the symbol $\boldsymbol{n}^{\mathrm{s}}$ stands for the unit normal vector on $\partial \Omega^{\mathrm{s}}$, the solid stress tensor is given by $\boldsymbol{\Pi}(\boldsymbol{d}, \dot{\boldsymbol{d}}) \stackrel{\text { def }}{=} \boldsymbol{\pi}(\boldsymbol{d})+\beta \boldsymbol{\pi}(\dot{\boldsymbol{d}})$, where $\boldsymbol{\pi}(\boldsymbol{d}) \stackrel{\text { def }}{=} 2 \lambda_{1} \varepsilon(\boldsymbol{d})+\lambda_{2}(\boldsymbol{\nabla}$. $\boldsymbol{d}) \boldsymbol{I}$ and $\lambda_{1}, \lambda_{2}$ denote the Lamé coefficients. Damping effects in the solid are thus modeled via the Rayleigh-like term $\alpha \rho^{\mathrm{s}} \dot{\boldsymbol{d}}-\beta \boldsymbol{\nabla} \cdot \boldsymbol{\pi}(\dot{\boldsymbol{d}})$.

The fundamental ingredient in the derivation of the schemes described in the previous sections is the interface Robin consistency (3) featured by the continuous problem (1)-(2). Unfortunately, this property is not shared by the coupled problem (1), (8) and (9), since the inertial term in (8) is distributed on the whole solid domain $\Omega^{\mathrm{s}}$ and $\Sigma \neq \Omega^{\mathrm{s}}$. The following generalized interface Robin consistency can however be recovered after discretization in space, using a lumped-mass approximation in the structure (see Fernández et al. [2015]):

$$
\boldsymbol{\sigma}(\boldsymbol{u}, p) \boldsymbol{n}+\rho^{\mathrm{s}} \boldsymbol{B}_{h} \partial_{t} \boldsymbol{u}=\rho^{\mathrm{s}} \boldsymbol{B}_{h} \partial_{t} \dot{\boldsymbol{d}}-\boldsymbol{\Pi}(\boldsymbol{d}, \dot{\boldsymbol{d}}) \boldsymbol{n}^{\mathrm{s}} \quad \text { on } \quad \Sigma .
$$

Note that, instead of the usual identity operator, the interface condition (10) involves the discrete interface operator $\boldsymbol{B}_{h}$, which consistently accounts for the solid inertial effects within the fluid. In fact, at the algebraic level, this operator is given by the interface entries of the solid lumped-mass matrix. Instead of formulating the time splitting in terms of (9), we consider (10) and $(9)_{2}$. This yields the following Robin-Neumann splitting of (9): 


$$
\left\{\begin{array}{r}
\boldsymbol{\sigma}\left(\boldsymbol{u}^{n}, p^{n}\right) \boldsymbol{n}+\frac{\rho^{\mathrm{s}}}{\tau} \boldsymbol{B}_{h} \boldsymbol{u}^{n}=\frac{\rho^{\mathrm{s}}}{\tau} \boldsymbol{B}_{h}\left(\dot{\boldsymbol{d}}^{n-1}+\tau \partial_{\tau} \dot{\boldsymbol{d}}^{\star}\right)-\boldsymbol{\Pi}\left(\boldsymbol{d}^{\star}, \dot{\boldsymbol{d}}^{\star}\right) \boldsymbol{n}^{\mathrm{s}}, \\
\boldsymbol{\Pi}\left(\boldsymbol{d}^{n}, \dot{\boldsymbol{d}}^{n}\right) \boldsymbol{n}^{\mathrm{s}}=-\boldsymbol{\sigma}\left(\boldsymbol{u}^{n}, p^{n}\right) \boldsymbol{n} .
\end{array}\right.
$$
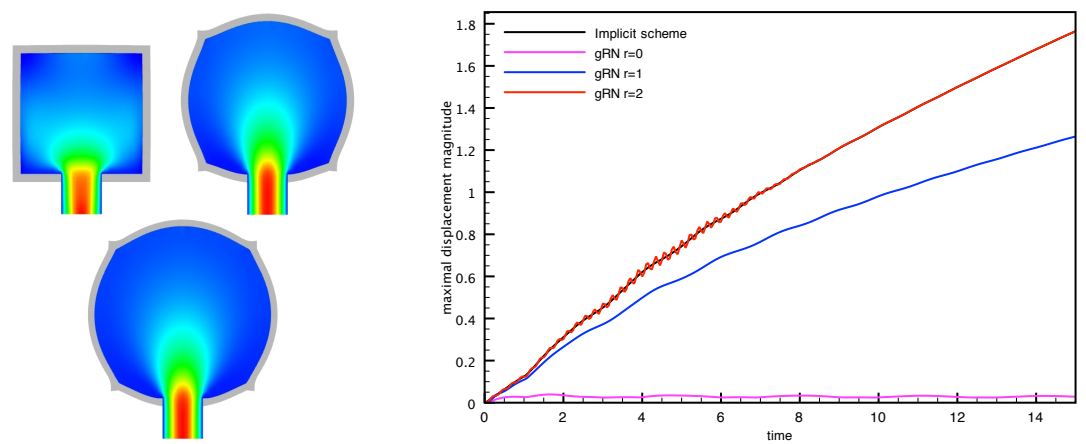

Fig. 4 Left: Snapshots of the fluid velocity magnitude in the deformed configurations at $t=0.15,7.5,15$ (generalized Robin-Neumann explicit coupling (11) with $r=1$ and $\tau=0.025)$. Right: Comparison of the solid displacements vs. time obtained with the generalized Robin-Neumann explicit coupling (11) and the implicit scheme $(\tau=0.025)$.

The analysis reported in Fernández et al. [2015] shows that the splitting (11) preserves the energy stability of the original Robin-Neumann explicit coupling paradimg (Algorithm 2). Numerical evidence indicates, however, that their optimal (first-order) accuracy is not preserved. Indeed, the order of the kinematic perturbation induced by the splitting (11) is expected to be $\mathcal{O}\left(\tau^{2^{r-1}} / h^{\frac{1}{2}}\right)$. Interestingly, the factor $h^{-\frac{1}{2}}$ is intrinsically related to the thick-walled character of the structure, through the non-uniformity of the discrete viscoelastic operator, and not to the mass lumping approximation (see Fernández and Mullaert [2015]).

We conclude this section by considering the balloon-like example of Section 3.1 but, this time, involving a thick-walled structure. In Figure 4(left) we have reported some snapshots of the fluid velocity magnitude and of the deformed configurations obtained with the generalized Robin-Neumann splitting (11) with $r=1$ and $\tau=0.025$. A comparison of the different variants with the implicit schemes is given in Figure 4(right). Note that spurious oscillations are visible for the explicit coupling with $r=2$. This is consistent with the fact that stability conditions are expected to be more restrictive in the case of the coupling with thick-walled structures. Considering that the value of $\tau$ is twice smaller than in Section 3.1, the poor accuracy of the explicit scheme with $r=0$ is even more striking. For $r=1$ and $r=2$ we obtain practically the same results as in Section 3.1. This is a clear indication of the $h^{-\frac{1}{2}}$ perturbation introduced by the splitting: the time-step length must be reduced to achieve a similar level of accuracy as in the thin-walled case. 


\section{Conclusion}

We have discussed a class of explicit coupling schemes for incompressible fluid-structure interaction. The key ingredient in the derivation of the methods is the notion of interface Robin consistency which depends on the thinor thick-walled character of the structure. In the case of the coupling with a thin-walled structure, energy stability and optimal first-order accuracy are retrieved without any restriction on the discretization parameters. Besides, under this structure regime, two promising extensions which deliver secondorder time-accuracy have been presented. The main issue regarding thickwalled structures is accuracy, since the perturbation induced by the splitting is not uniform with respect to the spatial discretization step $h$. It is worth noting, however, that the scheme with first-order extrapolation yields convergence under a standard hyperbolic-condition without the need of correction iterations.

Acknowledgements This work was supported by the French National Research Agency (ANR) through the EXIFSI project (ANR-12-JS01-0004)

\section{References}

S. Badia, F. Nobile, and C. Vergara. Fluid-structure partitioned procedures based on Robin transmission conditions. J. Comput. Phys., 227(14):70277051, 2008.

M. Bukač, S. Čanić, R. Glowinski, J. Tambača, and A. Quaini. Fluidstructure interaction in blood flow capturing non-zero longitudinal structure displacement. J. Comput. Phys., 235:515-541, 2013.

E. Burman and M.A. Fernández. Stabilization of explicit coupling in fluidstructure interaction involving fluid incompressibility. Comput. Methods Appl. Mech. Engrg., 198(5-8):766-784, 2009.

E. Burman and M.A. Fernández. Explicit strategies for incompressible fluidstructure interaction problems: Nitsche type mortaring versus Robin-Robin coupling. Int. J. Num. Meth. Engrg., 97(10):739-758, 2014.

P. Causin, J. F. Gerbeau, and F. Nobile. Added-mass effect in the design of partitioned algorithms for fluid-structure problems. Comput. Methods Appl. Mech. Engrg., 194(42-44):4506-4527, 2005.

D. Chapelle and K.J. Bathe. The finite element analysis of shellsfundamentals. Computational Fluid and Solid Mechanics. Springer-Verlag, Berlin, 2011.

M.A. Fernández. Coupling schemes for incompressible fluid-structure interaction: implicit, semi-implicit and explicit. SeMA J., (55):59-108, 2011. 
M.A. Fernández. Incremental displacement-correction schemes for incompressible fluid-structure interaction: stability and convergence analysis. $\mathrm{Nu}$ mer. Math., 123(1):21-65, 2013.

M.A. Fernández and J. Mullaert. Convergence analysis for a class of splitting schemes in incompressible fluid-structure interaction. Research Report RR8670, Inria, January 2015. URL https://hal.inria.fr/hal-01102975.

M.A. Fernández, J. Mullaert, and M. Vidrascu. Explicit Robin-Neumann schemes for the coupling of incompressible fluids with thin-walled structures. Comput. Methods Appl. Mech. Engrg., 267:566-593, 2013.

M.A. Fernández, J. Mullaert, and M. Vidrascu. Generalized Robin-Neumann explicit coupling schemes for incompressible fluid-structure interaction: stability analysis and numerics. Int. J. Num. Meth. Engrg., 101(3):199-229, 2015.

C. Förster, W.A. Wall, and E. Ramm. Artificial added mass instabilities in sequential staggered coupling of nonlinear structures and incompressible viscous flows. Comput. Methods Appl. Mech. Engrg., 196(7):1278-1293, 2007.

G. Guidoboni, R. Glowinski, N. Cavallini, and S. Canić. Stable looselycoupled-type algorithm for fluid-structure interaction in blood flow. $J$. Comput. Phys., 228(18):6916-6937, 2009.

M. Heil and A.L. Hazel. Fluid-structure interaction in internal physiological flows. Annu. Rev. Fluid Mech., 43:141-162, 2011.

P. Kalita and R. Schaefer. Mechanical models of artery walls. Arch. Comput. Methods Eng., 15(1):1-36, 2008.

U. Küttler, C. Förster, and W.A. Wall. A solution for the incompressibility dilemma in partitioned fluid-structure interaction with pure Dirichlet fluid domains. Comput. Mech., 38:417-429, 2006.

M. Lombardi, N. Parolini, A. Quarteroni, and G. Rozza. Numerical simulation of sailing boats: Dynamics, FSI, and shape optimization. In G. Buttazzo and A. Frediani, editors, Variational Analysis and Aerospace Engineering: Mathematical Challenges for Aerospace Design, Springer Optimization and Its Applications, pages 339-377. Springer, 2012.

M. Lukáčová-Medvid'ová, G. Rusnáková, and A. Hundertmark-Zaušková. Kinematic splitting algorithm for fluid-structure interaction in hemodynamics. Comput. Methods Appl. Mech. Engrg., 265(1):83-106, 2013.

P. Moireau, N. Xiao, M. Astorino, C. A. Figueroa, D. Chapelle, C. A. Taylor, and J-F. Gerbeau. External tissue support and fluid-structure simulation in blood flows. Biomech. Model. Mechanobiol., 11:1-18, 2012.

M.P. Païdoussis, S.J. Price, and E. de Langre. Fluid-structure interactions: cross-flow-induced instabilities. Cambridge University Press, 2011.

K. Takizawa and T.E. Tezduyar. Computational methods for parachute fluidstructure interactions. Arch. Comput. Methods Eng., 19:125-169, 2012.

D. Valdez-Jasso, H.T. Banks, M.A. Haider, D. Bia, Y. Zocalo, R.L. Armentano, and M.S. Olufsen. Viscoelastic models for passive arterial wall dynamics. Adv. Appl. Math. Mech., 1(2):151-165, 2009. 